\title{
Analytic Model Predictive Control of Uncertain Nonlinear Systems: A Fuzzy Adaptive Approach
}

\author{
Xiuyan Peng, ${ }^{1}$ Shuli Jia, ${ }^{1}$ and Xingmei Wang ${ }^{2}$ \\ ${ }^{1}$ College of Automation, Harbin Engineering University, Harbin 150001, China \\ ${ }^{2}$ Computer Science and Technology College, Harbin Engineering University, Harbin 150001, China \\ Correspondence should be addressed to Xiuyan Peng; jiashuli_1019@sina.com
}

Received 4 February 2015; Accepted 3 March 2015

Academic Editor: Xinggang Yan

Copyright (c) 2015 Xiuyan Peng et al. This is an open access article distributed under the Creative Commons Attribution License, which permits unrestricted use, distribution, and reproduction in any medium, provided the original work is properly cited.

\begin{abstract}
A fuzzy adaptive analytic model predictive control method is proposed in this paper for a class of uncertain nonlinear systems. Specifically, invoking the standard results from the Moore-Penrose inverse of matrix, the unmatched problem which exists commonly in input and output dimensions of systems is firstly solved. Then, recurring to analytic model predictive control law, combined with fuzzy adaptive approach, the fuzzy adaptive predictive controller synthesis for the underlying systems is developed. To further reduce the impact of fuzzy approximation error on the system and improve the robustness of the system, the robust compensation term is introduced. It is shown that by applying the fuzzy adaptive analytic model predictive controller the rudder roll stabilization system is ultimately uniformly bounded stabilized in the $H$-infinity sense. Finally, simulation results demonstrate the effectiveness of the proposed method.
\end{abstract}

\section{Introduction}

Currently, the study of nonlinear systems mainly includes feedback linearization method, backstepping, forwarding, and passivity method. Backstepping and passivity method apply for nonlinear systems with lower triangular structural features; forwarding method is suitable for systems with upper triangular structural features. These methods for nonlinear systems have a strict requirement in the system form. Feedback linearization method can turn the nonlinear system into a linear system, which is an effective solution to a nonlinear system control. However, analytic model predictive not only eliminates the restrictions of the nonlinear system form but also combines the advantages of predictive control and feedback linearization method; it can be seen as an optimized feedback linearization method applying predictive control thought. Compared with feedback linearization, it improves the control precision of the system. It is a control algorithm based on model optimization, with easy modeling, control performance, robustness, and simple logical structure and other characteristics, and has been widely used in recent years.
Literatures [1-3] propose the analytic model predictive control algorithm. In those papers, the predictive control in the literature requires that the controlled object is known, and the system uncertainties impose greater impact on predictive control. Thus, analytic model predictive control usually requires the controlled object to be precisely known. However, due to uncertainties of various disturbances, modelling errors, and so forth, in the actual project, we are often unable to obtain the accurate controlled plant model. Therefore, how to design a good control effect analytic model predictive control law in the case of uncertainties in system is crucial.

When the controlled object is known, many approaches are given by literatures [4-11] to solve this problem. But we hope to improve analytic model predictive control algorithm to complete control of the system, because the method can avoid the calculation of online optimization predictive control, thus saving the amount of computation and reducing the complexity of solving the problem. Considering that the fuzzy system has the ability to approximate the unknown nonlinear system function and uncertainties, applying the fuzzy system to control nonlinear uncertain systems has 
become a hot spot theory and engineering research and made a lot of research results (see [12-18]).

For solving the analytic model predictive control law which cannot be determined precisely because of system uncertainty, a fuzzy adaptive analytic model predictive control law is given in this paper based on adaptive fuzzy concept. Using fuzzy system to approximate the uncertainties in the controller, weights of fuzzy systems are based on system feedback error online adjustment, to make fuzzy system approach the unknown functions of controller. Secondly, considering the impacts of the fuzzy modeling errors on the system, the dynamic performance of the system is therefore reduced. A robust compensation term based on $H$-infinity method is introduced to eliminate this influence. According to Lyapunov stability theory, the closed-loop system ultimately bounded stable is proved.

Moreover, in those papers, the solved problem is that the dimensions of output are equal to the dimensions of input. But there were many situations in practical engineering in which the dimensions on input and output of system were not equal. Therefore, Moore-Penrose inverse of matrix is proposed in this paper; it can make the design process of the controller simple.

Rudder roll stabilization system as an emerging antirolling method attracts extensive attention domestically and abroad. The ships' motion environment and their movement characteristics show that it is difficult to get an accurate mathematical model. Model parameters uncertainty, unmodeled dynamics, and other issues increase the difficulty of determining rudder roll stabilization system control algorithm. The proposed method will be applied on rudder roll stabilization system; the simulation results show good effectiveness. The proposed method has important theoretical and practical value.

\section{Problem for Mulation}

A class of nonlinear system is given by

$$
\begin{aligned}
& \dot{\mathbf{x}}=\mathbf{f}(\mathbf{x})+\mathbf{g}(\mathbf{x}) \mathbf{u}, \\
& \mathbf{y}=\mathbf{h}(\mathbf{x}),
\end{aligned}
$$

where $\mathbf{x} \in R^{n}, \mathbf{u} \in R^{n_{1}}, \mathbf{y} \in R^{m}, \mathbf{f}(\mathbf{x}) \in R^{n}, \mathbf{g}(\mathbf{x}) \in R^{n \times n_{1}}$, $\mathbf{h}(\mathbf{x})=\left[\begin{array}{lll}h_{1}(x) & \cdots & h_{m}(x)\end{array}\right]^{T}$, and $n_{1} \leq m \leq n$.

If there exist uncertainties in $\mathbf{f}(\mathbf{x})$ and $\mathbf{g}(\mathbf{x})$, system (1) is a class of uncertain nonlinear systems.

The performance index adopted for system (1) is given by

$$
J=\frac{1}{2} \int_{0}^{T_{1}}\left(\widehat{\mathbf{y}}(\mathbf{t}+\boldsymbol{\tau})-\mathbf{y}_{\mathbf{d}}(\mathbf{t}+\boldsymbol{\tau})\right)^{\mathbf{T}}\left(\widehat{\mathbf{y}}(\mathbf{t}+\boldsymbol{\tau})-\mathbf{y}_{\mathbf{d}}(\mathbf{t}+\boldsymbol{\tau})\right) d \tau,
$$

where $\widehat{\mathbf{y}}(\mathbf{t}+\boldsymbol{\tau})$ is predictive value of output in the $\left[t, t+T_{1}\right], \tau \in$ $\left[0, T_{1}\right], T_{1}$ is the predictive period, and $\mathbf{y}_{\mathbf{d}}(\mathbf{t}+\boldsymbol{\tau})$ is reference signal in the $\left[t, t+T_{1}\right]$.

The following assumptions are imposed on nonlinear system (1) (see [2]).

(1) Each of the system outputs $\mathbf{y}(\mathbf{t})$ has the same relative degree $\rho$ and the zero dynamics are stable.
(2) All states are available.

(3) The output $\mathbf{y}(\mathbf{t})$ and the reference signal $\mathbf{y}_{\mathbf{d}}(\mathbf{t})$ are sufficiently many times continuously differentiable with respect to $t$.

If $\mathbf{f}(\mathbf{x})$ and $\mathbf{g}(\mathbf{x})$ are known, the control order of $\mathbf{u}$ is selected 1. Similarly, the higher derivatives of the output $\widehat{\mathbf{y}}(\mathbf{t})$, seeking $\boldsymbol{\rho}+\mathbf{l}$ order derivative, are denoted by $\widehat{\mathbf{Y}}(\mathbf{t})$ :

$$
\widehat{\mathbf{Y}}(\mathbf{t})=\left[\begin{array}{c}
\widehat{\mathbf{y}}(\mathbf{t}) \\
\widehat{\mathbf{y}}^{(\mathbf{1})}(\mathbf{t}) \\
\mathbf{M} \\
\widehat{\mathbf{y}}^{(\rho)}(\mathbf{t}) \\
\widehat{\mathbf{y}}^{(\rho+1)}(\mathbf{t}) \\
\mathbf{M} \\
\mathbf{y}^{(\rho+1)}(\mathbf{t})
\end{array}\right]=\left[\begin{array}{c}
\mathbf{h}(\mathbf{x}) \\
\mathrm{L}_{\mathbf{f}} \mathbf{h}(\mathbf{x}) \\
\mathbf{M} \\
\mathbf{L}_{\mathbf{f}}^{\rho} \mathbf{h}(\mathbf{x}) \\
\mathbf{L}_{\mathbf{f}}^{\rho+\mathbf{1}} \mathbf{h}(\mathbf{x}) \\
\mathbf{M} \\
\mathrm{L}_{\mathbf{f}}^{\rho+1} \mathbf{h}(\mathbf{x})
\end{array}\right]+\left[\begin{array}{c}
\mathbf{0}_{\rho \times \mathbf{1}} \\
\mathbf{L}(\widehat{\overline{\mathbf{u}}})
\end{array}\right],
$$

where $\boldsymbol{\rho}$ is the relative degree of system (1), $\mathbf{l}$ is control order, and $\widehat{\overline{\mathbf{u}}}=\left[\widehat{\mathbf{u}}(\mathbf{t}), \dot{\widehat{\mathbf{u}}}(\mathbf{t}), \ldots, \widehat{\mathbf{u}}^{[\mathbf{l}-\boldsymbol{\rho}]}(\mathbf{t})\right]^{T}$. Consider

$$
L(\widehat{\overline{\mathbf{u}}})=\left[\begin{array}{c}
\mathbf{L}_{\mathbf{g}} \mathbf{L}_{\mathbf{f}}^{\rho-1} \mathbf{h}(\mathbf{x}) \widehat{\mathbf{u}}(\mathbf{t}) \\
\mathbf{L}_{\mathbf{g}} \mathbf{L}_{\mathbf{f}}^{\rho-1} \mathbf{h}(\mathbf{x}) \dot{\hat{\mathbf{u}}}(\mathbf{t})+\mathbf{p}_{\mathbf{1 1}}(\widehat{\mathbf{u}}(\mathbf{t}), \mathbf{x}(\mathbf{t})) \\
\vdots \\
\mathbf{L}_{\mathbf{g}} \mathbf{L}_{\mathbf{f}}^{\rho-1} \mathbf{h}(\mathbf{x}) \widehat{\mathbf{u}}^{[\mathbf{l}]}(\mathbf{t})+\mathbf{p}_{\mathbf{r} \mathbf{1}}(\widehat{\mathbf{u}}(\mathbf{t}), \mathbf{x}(\mathbf{t})) \\
+\mathbf{L}_{\mathbf{r r}}\left(\widehat{\mathbf{u}}(\mathbf{t}), \dot{\hat{\mathbf{u}}}(\mathbf{t}), \mathbf{L}, \widehat{\mathbf{u}}^{[\mathbf{r}-1]}(\mathbf{t}), \mathbf{x}(\mathbf{t})\right)
\end{array}\right]
$$

where $r=\mathbf{1}-\boldsymbol{\rho}$ :

$$
\begin{aligned}
\mathbf{p}_{11}(\widehat{\mathbf{u}}(\mathbf{t}), \mathbf{x}(\mathbf{t})) & \\
= & \mathbf{L}_{\mathbf{g}} \mathbf{L}_{\mathbf{f}}^{\rho} \mathbf{h}(\mathbf{x}) \widehat{\mathbf{u}}(\mathbf{t}) \\
& +\frac{\partial\left(\mathbf{L}_{\mathbf{g}} \mathbf{L}_{\mathbf{f}}^{\rho-1} \mathbf{h}(\mathbf{x}) \widehat{\mathbf{u}}(\mathbf{t})\right)}{\partial \mathbf{x}}(\mathbf{f}(\mathbf{x})+\mathbf{g}(\mathbf{x}) \widehat{\mathbf{u}}(\mathbf{t})) .
\end{aligned}
$$

Within the moving time frame, the output $\widehat{\mathbf{y}}(\mathbf{t}+\boldsymbol{\tau})$ and $\mathbf{y}_{\mathbf{d}}(\mathbf{t}+\boldsymbol{\tau})$ at the time $\boldsymbol{\tau}$ is approximately predicted by

$$
\begin{aligned}
\widehat{\mathbf{y}}(\mathbf{t}+\boldsymbol{\tau}) & =\Gamma(\tau) \widehat{\mathbf{Y}}(\mathbf{t}), \\
\mathbf{y}_{\mathbf{d}}(\mathbf{t}+\boldsymbol{\tau}) & =\Gamma(\tau) \mathbf{Y}_{\mathbf{d}}(\mathbf{t}),
\end{aligned}
$$

where

$$
\begin{gathered}
\Gamma(\boldsymbol{\tau})=\left[1, \tau, \ldots, \frac{\tau^{\rho+l}}{(\rho+l) !}\right] \\
\mathbf{Y}_{\mathbf{d}}(\mathbf{t})=\left[\mathbf{y}_{\mathbf{d}}(\mathbf{t}), \dot{\mathbf{y}}_{\mathbf{d}}(\mathbf{t}), \ldots, \mathbf{y}_{\mathbf{d}}^{(\rho+\mathbf{l})}(\mathbf{t})\right]^{T} .
\end{gathered}
$$

Take (7) and (8) into the performance function (2):

$$
J=\frac{1}{2}\left[\widehat{\mathbf{Y}}(\mathbf{t})-\mathbf{Y}_{\mathbf{d}}(\mathbf{t})\right]^{T}\left[\widehat{\mathbf{Y}}(\mathbf{t})-\mathbf{Y}_{\mathbf{d}}(\mathbf{t})\right] \int_{0}^{T_{1}} \boldsymbol{\Gamma}(\boldsymbol{\tau})^{\mathbf{T}} \boldsymbol{\Gamma}(\boldsymbol{\tau}) d \tau .
$$


So the analytic model predictive control law of system (1) can be given by [1]

$$
\mathbf{G}(\mathbf{x}) \mathbf{u}+\mathbf{F}(\mathbf{x})+\mathbf{K M}_{\rho}-\mathbf{Y}_{\mathbf{d}}^{\rho}=\mathbf{0},
$$

where

$$
\begin{aligned}
& \mathbf{F}(\mathbf{x})=\left[\begin{array}{c}
L_{\mathbf{f}}^{\rho} h_{1}(\mathbf{x}) \\
\vdots \\
L_{\mathbf{f}}^{\rho} h_{m}(\mathbf{x})
\end{array}\right]_{m \times 1}, \\
& \mathbf{M}_{\boldsymbol{\rho}}=\left[\begin{array}{c}
\mathbf{y}(\mathbf{x})-\mathbf{y}_{\mathbf{d}}(\mathbf{x}) \\
\dot{\mathbf{y}}(\mathbf{x})-\dot{\mathbf{y}}_{\mathbf{d}}(\mathbf{x}) \\
\vdots \\
\mathbf{L}_{\mathbf{f}}^{\rho-1} \mathbf{h}(\mathbf{x})-\mathbf{y}_{\mathbf{d}}^{\rho-1}(\mathbf{x})
\end{array}\right]_{m \rho \times 1}, \\
& \mathbf{G}(\mathbf{x})=\left[\begin{array}{ccc}
L_{g_{1}} L_{\mathbf{f}}^{\rho-1} h_{1}(\mathbf{x}) & \cdots & L_{g_{n_{1}}} L_{\mathbf{f}}^{\rho-1} h_{1}(\mathbf{x}) \\
L_{g_{1}} L_{\mathbf{f}}^{\rho-1} h_{2}(\mathbf{x}) & \cdots & L_{g_{n_{1}}} L_{\mathbf{f}}^{\rho-1} h_{2}(\mathbf{x}) \\
\vdots & \ddots & \vdots \\
L_{g_{1}} L_{\mathbf{f}}^{\rho-1} h_{m}(\mathbf{x}) & \cdots & L_{g_{n_{1}}} L_{\mathbf{f}}^{\rho-1} h_{m}(\mathbf{x})
\end{array}\right]_{m \times n_{1}}
\end{aligned}
$$

Because of $n_{1} \leq m$, if $n_{1}=m$, the control law can be improved as follows:

$$
\mathbf{u}=-\mathbf{G}(\mathbf{x})^{-1}\left[\mathbf{F}(\mathbf{x})+\mathbf{K M}_{\boldsymbol{\rho}}-\mathbf{Y}_{\mathbf{d}}^{\rho}\right] .
$$

But if $n_{1}<m, \mathbf{G}(\mathbf{x})^{-1}$ does not exist, so (13) does not describe the control law of system (1).

In order to deal with this problem, we give a new definition.

Definition 1. When $G \in R^{m \times n}, G^{+} \in R^{n \times m} ; G^{+}$is said to be the Moore-Penrose inverse of $G$, if $G^{+}$can satisfy the Penrose conditions (see [19]):

(i) $G G^{+} G=G$;

(ii) $G^{+} G G^{+}=G^{+}$;

(iii) $\left(G G^{+}\right)^{H}=G G^{+}$;

(iv) $\left(G^{+} G\right)^{H}=G^{+} G$,

where $(\cdot)^{H}$ means conjugate transpose of matrix.

So the control law can be improved as follows when $n_{1}<$ $m$ :

$$
\mathbf{u}=-(\mathbf{G}(\mathbf{x}))^{+}\left[\mathbf{F}(\mathbf{x})+\mathbf{K M}_{\boldsymbol{\rho}}-\mathbf{Y}_{\mathbf{d}}^{\rho}\right],
$$

where $\mathbf{G}(\mathbf{x})^{+} \in R^{n_{1} \times m}$ is defined as Moore-Penrose inverse of $\mathbf{G}(\mathbf{x})$ and formula (14) is the minimum norm least-squares solution of formula (11) (see [19], for minimal norm leastsquares solution).

Remark 2. If the Moore-Penrose inverse of $\mathbf{G}(\mathbf{x})$ is used for formula (14), it can solve the problems of nonlinear system that the dimension of input and output cannot be necessarily equal. This is an expansion [1] in this paper, and it is more favorable for engineering applications.
Remark 3. When the Moore-Penrose inverse is used for formula (14), minimal norm least-squares solution for formula (11) can be obtained; it means that formula (14) is the minimum norm solution of (11), when $-\mathbf{G}(\mathbf{x}) \mathbf{u}=\mathbf{F}(\mathbf{x})+$ $\mathbf{K M}_{\rho}-\mathbf{Y}_{\mathbf{d}}^{\rho}$ is compatibility linear equation; if $-\mathbf{G}(\mathbf{x}) \mathbf{u}=\mathbf{F}(\mathbf{x})+$ $\mathbf{K M}_{\rho}-\mathbf{Y}_{\mathbf{d}}^{\rho}$ is incompatible linear equation, formula (14) is the least-squares solution of formula (11).

$\mathbf{K} \in R^{m \times m p}$ are elements of the front $m$ row of the matrix $\Gamma_{11}^{-1} \Gamma_{\rho 1}^{\mathrm{T}}$ :

$$
\begin{gathered}
\Gamma_{\mathbf{l l}}=\left(\begin{array}{ccc}
\Gamma_{(\rho+1, \rho+1)} & \cdots & \Gamma_{(\rho+1, \rho+1)} \\
\vdots & \ddots & \vdots \\
\Gamma_{(\rho+l+1, \rho+1)} & \cdots & \Gamma_{(\rho+l+1, \rho+l+1)}
\end{array}\right)_{(l+1) \times(l+1)}, \\
\Gamma_{\boldsymbol{\rho l}}=\left(\begin{array}{ccc}
\Gamma_{(1, \rho+1)} & \cdots & \Gamma_{(1, \rho+l+1)} \\
\vdots & \ddots & \vdots \\
\Gamma_{(\rho, \rho+1)} & \cdots & \Gamma_{(\rho, \rho+l+1)}
\end{array}\right)_{\rho \times(l+1)}, \\
\Gamma_{(i, j)}=\frac{T_{1}^{i+j-1}}{(i-1) !(j-1) !(i+j-1)}, \quad i, j=1, \ldots, \rho+l+1 .
\end{gathered}
$$

By the analytic model predictive control law (14) and its calculation, in order to get the control law of system (1), the $\mathbf{f}(\mathbf{x})$ and $\mathbf{g}(\mathbf{x})$ are known; otherwise $\mathbf{F}(\mathbf{x})$ and $\mathbf{G}(\mathbf{x})$ of predictive control law equation (14) cannot be accurately determined. When $\mathbf{f}(\mathbf{x})$ and $\mathbf{g}(\mathbf{x})$ are unknown, the controller will be unable to accurately calculate, so control performance will be affected. To solve this problem, we propose a fuzzy adaptive analytical model predictive controller.

\section{Controller Design}

3.1. Fuzzy Adaptive Analytical Model Predictive Controller Design. Fuzzy controller uses IF-THEN rules of fuzzy logic system, which uses a single point of obfuscation, product inference, and center of gravity defuzzification. $i$-rule is as follows.

$r^{i}$ : if $x_{1}$ is $A_{1}^{i}, x_{2}$ is $A_{2}^{i}$, and $\ldots$ and $x_{n}$ is $A_{n}^{i}$, then $\mathbf{y}$ is $\boldsymbol{\theta}_{i}, i=1, \ldots, L$, where $L$ is the number of rules for fuzzy systems.

Define the output of fuzzy system:

$$
\mathbf{y}=\boldsymbol{\theta}^{\mathrm{T}} \boldsymbol{\xi}(\mathbf{x})
$$

where $\boldsymbol{\theta}=\left[\theta_{1}, \ldots, \theta_{r}\right]$ is the weight coefficient that can be adjusted and $\boldsymbol{\xi}(\mathbf{x})=\left[\begin{array}{lll}\xi_{1}(\mathbf{x}) & \cdots & \xi_{L}(\mathbf{x})\end{array}\right]$ is fuzzy basis function:

$$
\xi_{i}(\mathbf{x})=\frac{\prod_{j=1}^{n} \mu_{a_{j}^{i}}\left(x_{j}\right)}{\sum_{i=1}^{r}\left[\prod_{j=1}^{n} \mu_{a_{j}^{i}}\left(x_{j}\right)\right]},
$$

where $\mu_{a_{j}^{i}}\left(x_{j}\right)$ is the membership function of the fuzzy system. 
If the controlled object exists uncertainty or unknown function, $\mathbf{F}(\mathbf{x})$ and $\mathbf{G}(\mathbf{x})$ are unknown; then the predictive control law equation (14) cannot be accurately determined, so we propose using $\widehat{\mathbf{F}}\left(\mathbf{x} \mid \boldsymbol{\theta}_{\mathrm{F}}\right)$ and $\widehat{\mathbf{G}}\left(\mathbf{x} \mid \boldsymbol{\theta}_{\mathrm{G}}\right)$ of fuzzy system to approach $\mathbf{F}(\mathbf{x})$ and $\mathbf{G}(\mathbf{x})$, respectively:

$$
\begin{aligned}
& \widehat{\mathbf{F}}\left(\mathbf{x} \mid \boldsymbol{\theta}_{\mathrm{F}}\right)=\boldsymbol{\theta}_{\mathrm{F}}^{\mathrm{T}} \xi_{\mathrm{F}}(\mathbf{x}), \\
& \widehat{\mathbf{G}}\left(\mathbf{x} \mid \boldsymbol{\theta}_{\mathrm{G}}\right)=\boldsymbol{\theta}_{\mathrm{G}}^{\mathrm{T}} \xi_{\mathrm{G}}(\mathbf{x}),
\end{aligned}
$$

where $\boldsymbol{\xi}_{\mathbf{F}}(\mathbf{x}) \in R^{L \times 1}$ and $\boldsymbol{\xi}_{\mathbf{G}}(\mathbf{x}) \in R^{L \times n_{1}}$.

Assumption 4. There exist optimal parameter vectors $\boldsymbol{\theta}_{\mathrm{F}}^{*}$ and $\boldsymbol{\theta}_{\mathrm{G}}^{*}$ of $\boldsymbol{\theta}_{\mathrm{F}}$ and $\boldsymbol{\theta}_{\mathrm{G}}$, which can make the output of fuzzy system approach $\mathbf{F}(\mathbf{x})$ and $\mathbf{G}(\mathbf{x})$ arbitrary precision:

$$
\begin{aligned}
& \boldsymbol{\theta}_{\mathbf{F}}^{*}=\underset{\boldsymbol{\theta}_{\mathbf{F}} \in \mathbf{M}_{\boldsymbol{F}_{\mathrm{F}}}}{\operatorname{argmin}}\left[\sup _{\mathbf{x} \in \mathbf{M}_{\mathbf{x}}}\left|\mathbf{F}(\mathbf{x})-\widehat{\mathbf{F}}\left(\mathbf{x} \mid \boldsymbol{\theta}_{\mathbf{F}}\right)\right|\right], \\
& \boldsymbol{\theta}_{\mathbf{G}}^{*}=\underset{\boldsymbol{\theta}_{\mathbf{G}} \in \mathbf{M}_{\boldsymbol{\theta}_{\mathbf{G}}}}{\operatorname{argmin}}\left[\sup _{\mathbf{x} \in \mathbf{M}_{\mathbf{x}}}\left|\mathbf{G}(\mathbf{x})-\widehat{\mathbf{G}}\left(\mathbf{x} \mid \boldsymbol{\theta}_{\mathbf{G}}\right)\right|\right] .
\end{aligned}
$$

Define the approximation error of fuzzy system:

$$
\boldsymbol{\omega}=\left[\widehat{\mathbf{F}}\left(\mathbf{x} \mid \boldsymbol{\theta}_{\mathrm{F}}\right)-\widehat{\mathbf{F}}\left(\mathbf{x} \mid \boldsymbol{\theta}_{\mathrm{F}}^{*}\right)\right]+\left[\widehat{\mathbf{G}}\left(\mathbf{x} \mid \boldsymbol{\theta}_{\mathbf{G}}\right)-\widehat{\mathbf{G}}\left(\mathbf{x} \mid \boldsymbol{\theta}_{\mathbf{G}}^{*}\right)\right] \mathbf{u} .
$$

The equivalent fuzzy predictive controller of (14) is given by

$$
\mathbf{u}_{\mathbf{p}}=-\widehat{\mathbf{G}}^{+}\left(\mathbf{x} \mid \boldsymbol{\theta}_{\mathrm{g}}\right)\left[\widehat{\mathbf{F}}\left(\mathbf{x} \mid \boldsymbol{\theta}_{\mathbf{f}}\right)+\mathbf{K M}_{\rho}-\mathbf{Y}_{\mathbf{d}}^{\rho}\right],
$$

where $\widehat{\mathbf{G}}^{+}\left(\mathbf{x} \mid \boldsymbol{\theta}_{\mathbf{g}}\right)$ is Moore-Penrose inverse of $\widehat{\mathbf{G}}\left(\mathbf{x} \mid \boldsymbol{\theta}_{\mathbf{g}}\right)$.

In order to overcome the impact of the approximation error $\boldsymbol{\omega}$ on system, it is necessary to introduce a robust compensation term $\mathbf{u}_{\mathrm{a}}$ to improve the performance of the whole system.

So the new control law is given by

$$
\begin{gathered}
\mathbf{u}_{\mathrm{c}}=-\widehat{\mathbf{G}}^{+}\left(\mathbf{x} \mid \boldsymbol{\theta}_{\mathrm{g}}\right)\left[\widehat{\mathbf{F}}\left(\mathbf{x} \mid \boldsymbol{\theta}_{\mathbf{f}}\right)+\mathbf{K M}_{\boldsymbol{\rho}}-\mathbf{Y}_{\mathbf{d}}^{\rho}+\mathbf{u}_{\mathrm{a}}\right], \\
\mathbf{u}_{\mathrm{a}}=\lambda_{a} \mathbf{B}^{\mathrm{T}} \mathbf{P} \widehat{\mathbf{e}},
\end{gathered}
$$

where the matrices $\mathbf{P}, \mathbf{B}$, and $\overline{\mathbf{e}}$ and parameter $\lambda_{a}$ are given in Section 3.2.

The adaptive law of $\boldsymbol{\theta}_{\mathrm{F}}, \boldsymbol{\theta}_{\mathrm{G}}$ is given by

$$
\begin{gathered}
\dot{\theta}_{\mathbf{F}}=\lambda_{F} \xi_{\mathbf{F}}(\mathbf{x}) \widehat{\mathbf{e}}^{\mathrm{T}} \mathbf{P B}, \\
\dot{\boldsymbol{\theta}}_{\mathbf{G}}=\lambda_{G} \xi_{\mathbf{G}}(\mathbf{x}) \mathbf{u}_{\mathbf{c}} \widehat{e}^{\mathrm{T}} \mathbf{P B},
\end{gathered}
$$

where $\lambda_{F}, \lambda_{G}$ are adaptive parameters of fuzzy system.

\subsection{Stability Analysis and Proof}

Theorem 5. If uncertain nonlinear system (1) chooses control law (24) and the adaptive law of formula (26), it can be satisfied: (i) closed-loop system is uniformly ultimately bounded;

(ii) for a given inhibition level $\gamma>0$, output tracking error can achieve $H_{\infty}$ performance:

$$
\begin{aligned}
\frac{1}{2} \int_{0}^{T} & \widehat{\mathbf{e}}^{\mathrm{T}} \mathbf{Q} \widehat{\mathbf{e}} d t \\
\leq & \frac{1}{2} \widehat{\mathbf{e}}^{\mathrm{T}}(\mathbf{0}) \mathbf{P} \widehat{\mathbf{e}}+\frac{1}{2} \operatorname{tr}\left(\widetilde{\boldsymbol{\theta}}_{\mathbf{F}}^{\mathrm{T}}(\mathbf{0}) \lambda_{F}^{-1} \widetilde{\boldsymbol{\theta}}_{\mathbf{F}}(\mathbf{0})\right) \\
& +\frac{1}{2} \operatorname{tr}\left(\widetilde{\boldsymbol{\theta}}_{\mathrm{G}}^{\mathrm{T}}(\mathbf{0}) \lambda_{G}^{-1} \widetilde{\boldsymbol{\theta}}_{\mathrm{G}}(\mathbf{0})\right)+\frac{1}{2} \gamma^{2} \int_{0}^{T}\|\boldsymbol{\omega}\|^{2} .
\end{aligned}
$$

Proof. $\widehat{\mathbf{y}}^{(\rho)}$ of formula (4) is given by

$$
\begin{aligned}
\widehat{\mathbf{y}}^{(\rho)}= & \mathbf{L}_{\mathbf{f}}^{\rho} \mathbf{h}(\mathbf{x})+\mathbf{L}_{\mathbf{g}} \mathbf{L}_{\mathbf{f}}^{\rho-1} \mathbf{h}(\mathbf{x}) \widehat{\mathbf{u}}(\mathbf{t}) \\
= & \mathbf{F}(\mathbf{x})+\mathbf{G}(\mathbf{x}) \widehat{\mathbf{u}}(\mathbf{t}) \\
= & \mathbf{F}(\mathbf{x})-\widehat{\mathbf{F}}\left(\mathbf{x} \mid \boldsymbol{\theta}_{\mathbf{F}}\right)+\left[\mathbf{G}(\mathbf{x})-\widehat{\mathbf{G}}\left(\mathbf{x} \mid \boldsymbol{\theta}_{\mathbf{G}}\right)\right] \widehat{\mathbf{u}}(\mathbf{t}) \\
& +\widehat{\mathbf{F}}\left(\mathbf{x} \mid \boldsymbol{\theta}_{\mathbf{F}}\right)+\widehat{\mathbf{G}}\left(\mathbf{x} \mid \boldsymbol{\theta}_{\mathbf{G}}\right) \widehat{\mathbf{u}}(\mathbf{t}) .
\end{aligned}
$$

Take (24) into (28):

$$
\begin{aligned}
\widehat{\mathbf{y}}^{(\rho)}= & \mathbf{F}(\mathbf{x})-\widehat{\mathbf{F}}\left(\mathbf{x} \mid \boldsymbol{\theta}_{\mathbf{F}}\right)+\left[\mathbf{G}(\mathbf{x})-\widehat{\mathbf{G}}\left(\mathbf{x} \mid \boldsymbol{\theta}_{\mathbf{G}}\right)\right] \mathbf{u}_{\mathbf{c}}(\mathbf{t}) \\
& +\widehat{\mathbf{F}}\left(\mathbf{x} \mid \boldsymbol{\theta}_{\mathbf{F}}\right)+\widehat{\mathbf{G}}\left(\mathbf{x} \mid \boldsymbol{\theta}_{\mathbf{G}}\right) \mathbf{u}_{\mathbf{c}}(\mathbf{t}) .
\end{aligned}
$$

Take (24) into (29):

$$
\begin{aligned}
\widehat{\mathbf{y}}^{(\rho)}= & \mathbf{F}(\mathbf{x})-\widehat{\mathbf{F}}\left(\mathbf{x} \mid \boldsymbol{\theta}_{\mathbf{F}}\right) \\
& +\left[\mathbf{G}(\mathbf{x})-\widehat{\mathbf{G}}\left(\mathbf{x} \mid \boldsymbol{\theta}_{\mathbf{G}}\right)\right] \mathbf{u}_{\mathbf{c}}(\mathbf{t})-\mathbf{K M}_{\boldsymbol{\rho}}+\mathbf{Y}_{\mathbf{d}}^{(\rho)}-\mathbf{u}_{\mathbf{a}} .
\end{aligned}
$$

It can be also written as

$$
\begin{aligned}
& \mathbf{K M}_{\rho}+\widehat{\mathbf{y}}^{(\rho)}-\mathbf{Y}_{\mathbf{d}}^{(\rho)} \\
& \quad=\mathbf{F}(\mathbf{x})-\widehat{\mathbf{F}}\left(\mathbf{x} \mid \boldsymbol{\theta}_{\mathrm{F}}\right)+\left[\mathbf{G}(\mathbf{x})-\widehat{\mathbf{G}}\left(\mathbf{x} \mid \boldsymbol{\theta}_{\mathbf{G}}\right)\right] \mathbf{u}_{\mathbf{c}}(\mathbf{t})-\mathbf{u}_{\mathbf{a}},
\end{aligned}
$$

where $\mathbf{M}_{\rho}=\left[\begin{array}{c}\mathbf{y}(\mathbf{x})-\mathbf{y}_{\mathrm{d}}(\mathbf{x}) \\ \dot{\mathbf{y}}(\mathbf{x})-\mathbf{y}_{\mathrm{d}}(\mathbf{x}) \\ \vdots \\ \mathbf{L}_{\mathrm{f}}^{\rho-1} \mathbf{h}(\mathbf{x})-\mathbf{y}_{\mathrm{d}}^{\rho-1}(\mathbf{x})\end{array}\right]$.

So error equation is obtained that the $i$ th error equation is given by

$$
\begin{aligned}
e_{i}^{[\rho]} & +k_{i, \rho-1} e_{i}^{[\rho-1]}+\cdots+k_{i, 0} e_{i} \\
& =\mathbf{F}_{i}(\mathbf{x})-\widehat{\mathbf{F}}_{i}\left(\mathbf{x} \mid \boldsymbol{\theta}_{\mathbf{F}}\right)+\left[\mathbf{G}_{i}(\mathbf{x})-\widehat{\mathbf{G}}_{i}\left(\mathbf{x} \mid \boldsymbol{\theta}_{\mathbf{G}}\right)\right] \mathbf{u}_{\mathbf{c}}-\mathbf{u}_{\mathbf{a} i}
\end{aligned}
$$

where $k_{i, j}$ is the $i$ th row elements of the matrix $\mathbf{K}(i=$ $1, \ldots, m)$ and the $k_{i, j}$ is determined by the predictive period $T_{1}$, the relative degree $\rho$, and control order $l$.

So

$$
h(e)=e^{(\rho)}(t)+k_{i, \rho-1} e^{(\rho-1)}(t)+\cdots+k_{i, 0} e(t) .
$$


If we choose $k_{i, j}$ reasonable, it can make formula (33) as Hurwitz Polynomial.

Formula (33) can be also written as

$$
\dot{\overline{\mathbf{e}}}=\mathbf{A} \overline{\mathbf{e}}+\mathbf{B}\left(\widetilde{\boldsymbol{\theta}}_{\mathrm{F}}^{\mathrm{T}} \xi_{\mathrm{F}}(\mathbf{x})+\widetilde{\boldsymbol{\theta}}_{\mathrm{G}}^{\mathrm{T}} \xi_{\mathrm{G}}(\mathbf{x}) \mathbf{u}_{\mathrm{c}}+\boldsymbol{\omega}-\mathbf{u}_{\mathrm{a}}\right),
$$

where

$$
\begin{aligned}
& \widehat{\mathbf{e}}=\left[\widehat{\mathbf{e}}_{\mathbf{1}} \cdots \widehat{\mathbf{e}}_{\mathbf{m}}\right]^{T} \in R^{m \rho}, \\
& \widehat{\mathbf{e}}_{\mathbf{i}}=\left[e_{i} \cdots e_{i}^{\rho-1}\right]^{T} \text {, } \\
& \mathbf{A}=\operatorname{diag}\left(\mathbf{A}_{\mathbf{i}}, \ldots, \mathbf{A}_{\mathbf{m}}\right) \in R^{m \rho \times m \rho}, \\
& \mathbf{B}=\operatorname{diag}\left(\mathbf{B}_{\mathbf{i}}, \ldots, \mathbf{B}_{\mathbf{m}}\right) \in R^{m \rho \times m}, \\
& \mathbf{A}_{\mathbf{i}}=\left[\begin{array}{ccccc}
0 & 1 & 0 & \cdots & 0 \\
0 & 0 & 1 & \cdots & 0 \\
\vdots & \vdots & \vdots & \ddots & \vdots \\
-k_{i, 0} & -k_{i, 1} & -k_{i, 2} & \cdots & -k_{i, \rho-1}
\end{array}\right] \text {, } \\
& \mathbf{B}_{\mathbf{i}}=\left[\begin{array}{llll}
0 & 0 & \cdots & 1
\end{array}\right]^{T} \quad(i=1, \ldots, m), \\
& \widetilde{\boldsymbol{\theta}}_{\mathrm{F}}=\boldsymbol{\theta}_{\mathrm{F}}^{*}-\boldsymbol{\theta}_{\mathrm{F}}, \\
& \widetilde{\boldsymbol{\theta}}_{\mathrm{G}}=\boldsymbol{\theta}_{\mathrm{G}}^{*}-\boldsymbol{\theta}_{\mathrm{G}} \text {. }
\end{aligned}
$$

Lyapunov function is constructed as follows:

$$
\begin{aligned}
V & =V_{1}+V_{2}+V_{3}, \\
V_{1} & =\frac{1}{2} \widehat{\mathbf{e}}^{\mathbf{T}} \mathbf{P} \widehat{\mathbf{e}}, \\
V_{2} & =\frac{1}{2} \operatorname{tr}\left(\widetilde{\boldsymbol{\theta}}_{\mathbf{F}}^{\mathbf{T}} \lambda_{F}^{-1} \widetilde{\boldsymbol{\theta}}_{\mathbf{F}}\right), \\
V_{3} & =\frac{1}{2} \operatorname{tr}\left(\widetilde{\boldsymbol{\theta}}_{\mathbf{G}}^{\mathbf{T}} \lambda_{G}^{-1} \widetilde{\boldsymbol{\theta}}_{\mathbf{G}}\right),
\end{aligned}
$$

where tr represents trace of matrix.

$\mathbf{P}=\operatorname{diag}\left(\mathbf{P}_{\mathbf{1}}, \ldots, \mathbf{P}_{\mathbf{m}}\right) \in R^{m \rho \times m \rho}, \mathbf{P}_{\mathbf{i}}$ is positive symmetric matrix, and it satisfies the positive definite solution of Riccati equation:

$$
\begin{array}{r}
\mathbf{P}_{\mathbf{i}} \mathbf{A}_{\mathbf{i}}+\mathbf{A}_{\mathbf{i}}^{\mathrm{T}} \mathbf{P}_{\mathbf{i}}+\mathbf{Q}_{\mathbf{i}}-\left(\frac{2}{\lambda_{i}}-\frac{1}{\gamma^{2}}\right) \mathbf{P}_{\mathbf{i}} \mathbf{B}_{\mathbf{i}} \mathbf{B}_{\mathbf{i}}^{\mathrm{T}} \mathbf{P}_{\mathbf{i}}=\mathbf{0} \\
\left(\mathbf{Q}_{\mathbf{i}}=\mathbf{Q}_{\mathbf{i}}^{\mathrm{T}}>\mathbf{0}\right)
\end{array}
$$

where $\mathbf{Q}_{\mathbf{i}}$ is design parameter.

$V$ is derivative along the trajectory of the system:

$$
\dot{V}=\dot{V}_{1}+\dot{V}_{2}+\dot{V}_{3}
$$

where $\mathbf{M}=\mathbf{B}\left(\tilde{\boldsymbol{\theta}}_{\mathrm{F}}^{\mathrm{T}} \boldsymbol{\xi}_{\mathrm{F}}(\mathbf{x})+\tilde{\boldsymbol{\theta}}_{\mathrm{G}}^{\mathrm{T}} \boldsymbol{\xi}_{\mathrm{G}}(\mathbf{x}) \mathbf{u}_{\mathrm{c}}+\boldsymbol{\omega}-\mathbf{u}_{\mathbf{a}}\right)$ and

$$
\begin{aligned}
& \dot{V}_{1}=\frac{1}{2} \dot{\mathbf{e}} \mathbf{T} \mathbf{P} \widehat{\mathbf{e}}+\frac{1}{2} \widehat{\mathbf{e}}^{\mathrm{T}} \mathbf{P} \dot{\overrightarrow{\mathbf{e}}} \\
& =\frac{1}{2}\left(\widehat{\mathbf{e}}^{\mathrm{T}} \mathbf{A}^{\mathrm{T}} \mathbf{P} \widehat{\mathbf{e}}+\mathbf{M}^{\mathrm{T}} \mathbf{P} \widehat{\mathbf{e}}\right)+\frac{1}{2} \widehat{\mathbf{e}}^{\mathrm{T}} \mathbf{P A} \widehat{\mathbf{e}}+\frac{1}{2} \widehat{\mathbf{e}}^{\mathrm{T}} \mathbf{P} \mathbf{M} \\
& =\frac{1}{2} \widehat{\mathbf{e}}^{\mathrm{T}}\left(\mathbf{A}^{\mathrm{T}} \mathbf{P}+\mathbf{P A}\right) \widehat{\mathbf{e}}+\widehat{\mathbf{e}}^{\mathrm{T}} \mathbf{P} \mathbf{M} \\
& =-\frac{1}{2} \widehat{\mathbf{e}}^{\mathrm{T}} \mathbf{Q} \widehat{\mathbf{e}}+\frac{1}{2} \widehat{\mathbf{e}}^{\mathrm{T}}\left(\frac{2}{\lambda}-\frac{1}{\gamma^{2}}\right) \mathbf{P B B} \mathbf{B}^{\mathrm{T}} \mathbf{P} \widehat{\mathbf{e}} \\
& +\widetilde{\mathbf{e}}^{\mathrm{T}} \mathbf{P B}\left[\widetilde{\boldsymbol{\theta}}_{\mathrm{F}}^{\mathrm{T}} \xi_{\mathrm{F}}(\mathbf{x})+\widetilde{\boldsymbol{\theta}}_{\mathrm{G}}^{\mathrm{T}} \xi_{\mathrm{G}}(\mathbf{x}) \mathbf{u}+\boldsymbol{\omega}-\mathbf{u}_{\mathrm{a}}\right], \\
& \dot{V}_{2}=\lambda_{F}^{-1} \operatorname{tr}\left(\widetilde{\boldsymbol{\theta}}_{\mathbf{F}}^{\mathrm{T}} \dot{\tilde{\boldsymbol{\theta}}}_{\mathbf{F}}\right) \\
& =-\lambda_{F}^{-1} \operatorname{tr}\left(\widetilde{\boldsymbol{\theta}}_{\mathbf{F}}^{\mathrm{T}} \dot{\boldsymbol{\theta}}_{\mathbf{F}}\right) \text {, } \\
& \dot{V}_{3}=\lambda_{G}^{-1} \operatorname{tr}\left(\widetilde{\boldsymbol{\theta}}_{\mathrm{G}}^{\mathrm{T}} \dot{\tilde{\boldsymbol{\theta}}}_{\mathrm{G}}\right) \\
& =-\lambda_{G}^{-1} \operatorname{tr}\left(\widetilde{\boldsymbol{\theta}}_{\mathrm{G}}^{\mathrm{T}} \dot{\boldsymbol{\theta}}_{\mathrm{G}}\right) \text {. }
\end{aligned}
$$

So

$$
\begin{aligned}
\dot{V}= & -\frac{1}{2} \widehat{\mathbf{e}}^{\mathrm{T}} \mathbf{Q} \widehat{\mathbf{e}}+\widehat{\mathbf{e}}^{\mathrm{T}} \mathbf{P B}\left[\widetilde{\boldsymbol{\theta}}_{\mathrm{F}}^{\mathrm{T}} \xi_{\mathrm{F}}(\mathbf{x})+\widetilde{\boldsymbol{\theta}}_{\mathrm{G}}^{\mathrm{T}} \xi_{\mathrm{G}}(\mathbf{x}) \mathbf{u}_{\mathbf{c}}+\boldsymbol{\omega}-\mathbf{u}_{\mathrm{a}}\right] \\
& -\lambda_{F}^{-1} \operatorname{tr}\left(\widetilde{\boldsymbol{\theta}}_{\mathrm{F}}^{\mathrm{T}} \dot{\boldsymbol{\theta}}_{\mathrm{F}}\right)-\lambda_{G}^{-1} \operatorname{tr}\left(\widetilde{\boldsymbol{\theta}}_{\mathrm{G}}^{\mathrm{T}} \dot{\boldsymbol{\theta}}_{\mathrm{G}}\right) \\
= & -\frac{1}{2} \widehat{\mathbf{e}}^{\mathrm{T}} \mathbf{Q} \widehat{\mathbf{e}}+\left\{\widehat{\mathbf{e}}^{\mathrm{T}} \mathbf{P B} \widetilde{\boldsymbol{\theta}}_{\mathrm{F}}^{\mathrm{T}} \xi_{\mathrm{F}}(\mathbf{x})-\lambda_{F}^{-1} \operatorname{tr}\left(\widetilde{\boldsymbol{\theta}}_{\mathrm{F}}^{\mathrm{T}} \dot{\boldsymbol{\theta}}_{\mathrm{F}}\right)\right\} \\
& +\left\{\widehat{\mathbf{e}}^{\mathrm{T}} \mathbf{P B} \widetilde{\boldsymbol{\theta}}_{\mathrm{G}}^{\mathrm{T}} \xi_{\mathrm{G}}(\mathbf{x}) \mathbf{u}_{\mathbf{c}}-\lambda_{\mathrm{G}}^{-1} \operatorname{tr}\left(\widetilde{\boldsymbol{\theta}}_{\mathrm{G}}^{\mathrm{T}} \dot{\boldsymbol{\theta}}_{\mathrm{G}}\right)\right\} \\
& +\frac{1}{2} \widehat{\mathbf{e}}^{\mathrm{T}}\left(\frac{2}{\lambda}-\frac{1}{\gamma^{2}}\right) \mathbf{P B B} \mathbf{P B}^{\mathrm{T}} \mathbf{P} \widehat{\mathbf{e}}+\widehat{\mathbf{e}}^{\mathrm{T}} \mathbf{P B}\left\{\boldsymbol{\omega}-\mathbf{u}_{\mathbf{a}}\right\} .
\end{aligned}
$$

Because of $\dot{\boldsymbol{\theta}}_{\mathrm{F}}=\lambda_{F} \boldsymbol{\xi}_{\mathrm{F}}(\mathbf{x}) \widehat{\mathbf{e}}^{\mathrm{T}} \mathbf{P B}, \dot{\boldsymbol{\theta}}_{\mathbf{G}}=\lambda_{\mathrm{G}} \boldsymbol{\xi}_{\mathbf{G}}(\mathbf{x}) \mathbf{u}_{\mathbf{c}} \widehat{\mathbf{e}}^{\mathrm{T}} \mathbf{P B}$, $\mathbf{u}_{\mathbf{a}}=\lambda_{a} \mathbf{B}^{\mathrm{T}} \mathbf{P} \widehat{\mathbf{e}}$, and we choose $\lambda_{a}=1 / \lambda$.

Now it can obtain

$$
\begin{aligned}
\dot{V} & =-\frac{1}{2} \widehat{\mathbf{e}}^{\mathrm{T}} \mathbf{Q} \widehat{\mathbf{e}}+\widehat{\mathbf{e}}^{\mathrm{T}} \mathbf{P B} \boldsymbol{\omega}-\frac{1}{2 \gamma^{2}} \widehat{\mathbf{e}}^{\mathrm{T}} \mathbf{P B B}{ }^{\mathrm{T}} \mathbf{P} \widehat{\mathbf{e}}+\widehat{\mathbf{e}}^{\mathrm{T}} \mathbf{P B} \boldsymbol{\omega} \\
& =-\frac{1}{2} \widehat{\mathbf{e}}^{\mathrm{T}} \mathbf{Q} \widehat{\mathbf{e}}-\frac{1}{2}\left(\frac{1}{\gamma} \widehat{\mathbf{e}}^{\mathrm{T}} \mathbf{P B}-\gamma \boldsymbol{\omega}\right)^{2}+\frac{1}{2} \gamma^{2}\left\|\boldsymbol{\omega}^{2}\right\| \\
& \leq-\frac{1}{2} \widehat{\mathbf{e}}^{\mathrm{T}} \mathbf{Q} \widehat{\mathbf{e}}+\frac{1}{2} \gamma^{2}\|\boldsymbol{\omega}\|^{2} \\
& \leq-\frac{1}{2} \lambda_{\min }(\mathbf{Q})\|\widehat{\mathbf{e}}\|^{2}+\frac{1}{2} \gamma^{2}\|\overline{\boldsymbol{\omega}}\|^{2},
\end{aligned}
$$

where $\lambda_{\text {min }}(\mathbf{Q})$ is the smallest eigenvalues of matrix.

It can be known from (41), if

$$
\begin{gathered}
\|\mathbf{e}\| \geq \frac{\gamma\|\boldsymbol{\omega}\|}{\sqrt{\lambda_{\min }(\mathbf{Q})}}, \\
\dot{V} \leq 0 .
\end{gathered}
$$

So the closed-loop system is uniformly ultimately bounded. 
The integration of (41) from $t=0$ to $t=T$ is given by

$$
\frac{1}{2} \int_{0}^{\mathrm{T}} \widehat{\mathbf{e}}^{\mathrm{T}} \mathbf{Q} \overline{\mathbf{e}} d t \leq V(0)-V(T)+\frac{1}{2} \gamma^{2} \int_{0}^{\mathrm{T}}\|\boldsymbol{\omega}\|^{2} .
$$

Because of $V(T) \geq 0$,

$$
\begin{aligned}
\frac{1}{2} \int_{0}^{T} & \widehat{\mathbf{e}}^{\mathrm{T}} \mathbf{Q} \widehat{\mathbf{e}} d t \\
\leq & V(0)+\frac{1}{2} \gamma^{2} \int_{0}^{T}\|\omega\|^{2} \\
= & \frac{1}{2} \widehat{\mathbf{e}}^{\mathrm{T}}(\mathbf{0}) \mathbf{P} \widehat{\mathbf{e}}+\frac{1}{2} \operatorname{tr}\left(\widetilde{\boldsymbol{\theta}}_{\mathbf{F}}^{\mathbf{T}}(\mathbf{0}) \lambda_{\mathbf{F}}^{-1} \widetilde{\boldsymbol{\theta}}_{\mathbf{F}}(\mathbf{0})\right) \\
& +\frac{1}{2} \operatorname{tr}\left(\widetilde{\boldsymbol{\theta}}_{\mathbf{G}}^{\mathbf{T}}(\mathbf{0}) \lambda_{G}^{-1} \widetilde{\boldsymbol{\theta}}_{\mathbf{G}}(\mathbf{0})\right)+\frac{1}{2} \gamma^{2} \int_{0}^{T}\|\boldsymbol{\omega}\|^{2} .
\end{aligned}
$$

So output tracking error can achieve $H_{\infty}$ performance (27).

\section{Simulation Analysis}

In order to verify the control performance of the controller and engineering applications, apply it to rudder roll stabilization system. This paper is a transport ship (see [20]). The controlled object model is given by

$$
\begin{aligned}
& \dot{\mathbf{x}}=\mathbf{f}(\mathbf{x})+\Delta \mathbf{f}(\mathbf{x})+(\mathbf{g}(\mathbf{x})+\Delta \mathbf{g}(\mathbf{x})) \mathbf{u}+\mathbf{d}, \\
& \mathbf{y}=\mathbf{h}(\mathbf{x}),
\end{aligned}
$$

where $\Delta \mathbf{f}(\mathbf{x})$ and $\Delta \mathbf{g}(\mathbf{x})$ are unknown functions that are caused by changes of the speed and metacentric height; generally, these variations cannot be accurately determined. d is wave disturbance and its calculation is given by literature (see [20]).

If there are no unknown functions, it can be known:

$$
\begin{aligned}
& \dot{\mathbf{x}}=\mathbf{f}(\mathbf{x})+\mathbf{g}(\mathbf{x}) \mathbf{u}+\mathbf{d}, \\
& \mathbf{y}=\mathbf{h}(\mathbf{x}),
\end{aligned}
$$

where

$$
\begin{gathered}
\mathbf{x}=\left[x_{1}, x_{2}, x_{3}, x_{4}, x_{5}\right]^{T}=[v, p, r, \varphi, \psi]^{T}, \\
\mathbf{y}=\left[y_{1}, y_{2}\right]^{T}, \\
\mathbf{f}(\mathbf{x})=\left[f_{1}(x), f_{2}(x), f_{3}(x), f_{4}(x), f_{5}(x)\right]^{T}, \\
\mathbf{g}(\mathbf{x})=\left[b_{1}, b_{2}, b_{3}, 0,0\right]^{T}, \\
f_{1}(x)=a_{11} x_{1}+a_{12} x_{3}+a_{13} x_{1}\left|x_{1}\right|+a_{14} x_{1}\left|x_{3}\right|, \\
f_{2}(x)=a_{21} x_{2}+a_{22} x_{4}+a_{23} x_{4}^{3}, \\
f_{3}(x)=a_{31} x_{1}+a_{32} x_{3}+a_{33} x_{1} x_{3}, \\
f_{4}(x)=x_{2} ; \quad f_{5}(x)=x_{3},
\end{gathered}
$$

$$
\begin{gathered}
h_{1}(x)=x_{4} ; \quad h_{2}(x)=x_{5}, \\
a_{11}=-0.0833, \quad a_{12}=-1.6355, \\
a_{13}=-0.0215, \quad a_{14}=-0.6048, \\
a_{2}=-0.0763, \quad a_{22}=-0.3588, \quad a_{23}=0.7363 ; \\
a_{31}=-0.0028, \quad a_{32}=-0.2706, \quad a_{33}=-0.3091 ; \\
b_{1}=-0.2121, \quad b_{2}=0.0182, \quad b_{3}=0.0166,
\end{gathered}
$$

where $p, r, \varphi, \psi$ are roll velocity, yaw velocity, yaw, and roll angle, respectively.

The initial speed is $10 \mathrm{~m} / \mathrm{s}$; assume that $a_{i j}$ have perturbation of $15 \%$ and $b_{i}$ have perturbation of $20 \%$. In the simulation, simulate the variations $\Delta \mathbf{f}(\mathbf{x})$ and $\Delta \mathbf{g}(\mathbf{x})$ using a set of random numbers within the given range.

It can be calculated by the known conditions:

$$
\begin{aligned}
L_{f} h_{1}(x) & =\left[\begin{array}{llll}
0 & 0 & 1 & 0
\end{array}\right] \mathbf{f}=x_{1}, \\
L_{f} h_{2}(x) & =\left[\begin{array}{llll}
0 & 0 & 0 & 1
\end{array}\right] \mathbf{f}=x_{2}, \\
L_{f}^{2} h_{1}(x) & =\left[\begin{array}{llll}
1 & 0 & 0 & 0
\end{array}\right] \mathbf{f}=a_{22} x_{1}+a_{24} x_{3}+a_{29} x_{3}^{3}, \\
L_{f}^{2} h_{2}(x) & =\left[\begin{array}{llll}
0 & 1 & 0 & 0
\end{array}\right] \mathbf{f}=a_{32} x_{2}+a_{39} x_{2}^{2}, \\
L_{g} L_{f} h_{1}(x) & =\left[\begin{array}{llll}
1 & 0 & 0 & 0
\end{array}\right] \mathbf{g}=b_{1}, \\
L_{g} L_{f} h_{1}(x) & =\left[\begin{array}{llll}
0 & 1 & 0 & 0
\end{array}\right] \mathbf{g}=b_{2} .
\end{aligned}
$$

From (48), we know that $\rho_{1}=\rho_{2}=2$, so $\rho=4$; if we choose $\mathbf{y}_{\mathbf{d}}=\left[\begin{array}{c}0 \\ 20 / 57.3\end{array}\right], T_{1}=60 \mathrm{~s}$, and $l=1$, the $\mathbf{K}$ is calculated:

$$
\mathbf{K}=\left[\begin{array}{cccc}
0.05 & 0.32 & 0.90 & 1.33 \\
-0.03 & -0.22 & -0.55 & -0.61
\end{array}\right]
$$

We use the following fuzzy linguistic variables:

$A_{j}^{1}$ (negative large), $A_{j}^{2}$ (negative middle), $A_{j}^{3}$ (negative small), $A_{j}^{4}$ (zero), $A_{j}^{5}$ (positive small), $A_{j}^{6}$ (positive middle), and $A_{j}^{7}$ (positive large).

The membership functions are given by

$$
\mu_{a_{j}^{i}}\left(x_{j}\right)=\exp \left[-\left(x_{j}-\mu_{i}\right)^{2}\right],
$$

where $\mu_{i}=-0.5,-0.3,-0.1,0,0.1,0.3,0.5, i=1, \ldots, 7, j=$ 1,2 . This paper uses the seven fuzzy rules to approximate each variable of $F(x)$ and $G(x)$. The rules are as follows:

$$
\begin{aligned}
& r^{L}: \text { if } x_{1} \text { is } A_{1}^{i}, x_{2} \text { is } A_{2}^{i} \text {, and } \ldots \text { and } x_{n} \text { is } A_{n}^{i} \text {, then } y \text { is } \\
& F^{L}, i=1, \ldots, 7 .
\end{aligned}
$$

Remark 6. Because of single input and multiple outputs for system (45) in this paper, we choose $\boldsymbol{\xi}_{\mathrm{F}}(\mathbf{x})=\boldsymbol{\xi}_{\mathrm{G}}(\mathbf{x}) \in R^{L \times 1}$. The inverses of $\mathbf{G}(\mathbf{x})$ and $\widehat{\mathbf{G}}\left(\mathbf{x} \mid \boldsymbol{\theta}_{\mathbf{G}}\right)$ are used Moore-Penrose inverse. 
TABLE 1: Roll reduction rate and standard deviation of yaw when $h_{1 / 3}=4 \mathrm{~m}$.

\begin{tabular}{lcccc}
\hline Parameters & AP & RCS & RRR & CP \\
\hline i & 4.8626 & 1.8355 & $62.25 \%$ & 3.1008 \\
ii & 4.8682 & 2.3629 & $51.46 \%$ & 3.2504 \\
iii & 4.8682 & 1.9163 & $58.58 \%$ & 3.1723 \\
\hline
\end{tabular}

Initial weights values of fuzzy systems are given by

$$
\begin{gathered}
\boldsymbol{\theta}_{\mathbf{F} \mathbf{0}}=\mathbf{0}, \\
\boldsymbol{\theta}_{\mathbf{g} \mathbf{0}}=\mathbf{0}, \\
\mathbf{Q}=3 I_{8 \times 8}, \quad \lambda_{F}=10, \\
\lambda_{G}=2, \quad \lambda=1, \quad \gamma=5 .
\end{gathered}
$$

So $\lambda_{a}=1$.

The following simulation conditions and analysis of the simulation results to verify the effectiveness of the proposed method are given.

Condition 1. In system with the wave disturbance, wave period $T_{w}=8 \mathrm{~s}$, significant wave height $h_{1 / 3}=4 \mathrm{~m}$, and relative damping coefficient $\zeta=0.3$, encounter angle is $135^{\circ}$, a reference yaw angle is $20^{\circ}$, and wave disturbance calculation is given by [14].

(i) If there is no uncertain term in system (45), the control law is only analytic model predictive control for system simulation; the simulation results are shown in Figure 1.

(ii) If there are uncertain terms in system (45), the control law is only analytic model predictive control for system simulation; the simulation results are shown in Figure 2.

(iii) If there are uncertain terms in system (45), the control law is fuzzy adaptive analytic model predictive control for system simulation; the simulation results are shown in Figure 3.

Figures 1(a), 2(a), and 3(a) are output of roll angle; the dotted line is the ship rolling motion without adding the controller, and the solid line is the rolling motion using controller. Figures $1(b), 2(b)$, and $3(b)$ are the output of yaw angle.

In order to compare the effect of roll stabilization under different conditions, the roll reduction rate (RRR) is given by literature [12]:

$$
\operatorname{RRR}(\%)=\frac{\mathrm{AP}-\mathrm{RCS}}{\mathrm{AP}} \times 100 \%,
$$

where RCS and AP are the standard deviation of roll angle with and without the roll damping system. Assume that $\mathrm{CP}$ is standard deviation of yaw angle.

The roll reduction rate and standard deviation of yaw angle in each case can be seen in Table 1.

It is apparent that it achieves better tracking performance on fuzzy adaptive analytic model predictive control law and
TABLE 2: Roll reduction rate and standard deviation of yaw when $h_{1 / 3}=5.5 \mathrm{~m}$.

\begin{tabular}{lcccc}
\hline Parameters & AP & RCS & RRR & CP \\
\hline i & 7.4459 & 2.9333 & $60.61 \%$ & 3.1643 \\
ii & 7.4459 & 3.6529 & $50.94 \%$ & 3.3310 \\
iii & 7.4459 & 3.1594 & $57.57 \%$ & 3.2635 \\
\hline
\end{tabular}

the roll reduction rate achieves a satisfactory result when there are uncertain terms in system (45).

Condition 2. In a system with the wave disturbance, wave period $T_{w}=8.5 \mathrm{~s}$, significant wave height $h_{1 / 3}=5.5 \mathrm{~m}$, relative damping coefficient $\zeta=0.3$, encounter angle is $135^{\circ}$, and a reference yaw angle is $20^{\circ}$.

(i) If there is no uncertain term in system (45), the control law is only analytic model predictive control for system simulation; the simulation results are shown in Figure 4.

(ii) If there are uncertain terms in system (45), the control law is only analytic model predictive control for system simulation; the simulation results are shown in Figure 5.

(iii) If there are uncertain terms in system (45), the control law is fuzzy adaptive analytic model predictive control for system simulation; the simulation results are shown in Figure 6.

The roll reduction rate and standard deviation of yaw angle in each case can be seen in Table 2. As can be seen from Figure 5 when system (45) is uncertain, in dealing with wave disturbance strengthened, if we use only analytic model predictive control law, it will make the roll reduction rate significantly lower and part of roll angle will be increased. Figure 6 and Table 2 show that the proposed method in this paper has strong robustness.

The simulation results under Condition 2 are as shown in Figures 4, 5, and 6 and Table 2.

\section{Conclusions}

Robustness and control effect are important factors to measure the control quality of a nonlinear system. In the presence of uncertainty, the analytic model predictive control performance will be reduced significantly, and system cannot meet the robustness requirements. Using fuzzy system to approximate the uncertainties in the controller, weights of fuzzy systems are based on system feedback error online adjustment, to make fuzzy system approach the unknown functions of controller. Utilizing Taylor equation, the fuzzy adaptive predictive control law is achieved, and since online optimization is not required, the huge calculation burden of predictive control can be avoided. A robust compensation term is introduced to eliminate this influence which is the impact of the fuzzy modeling errors on the system. 

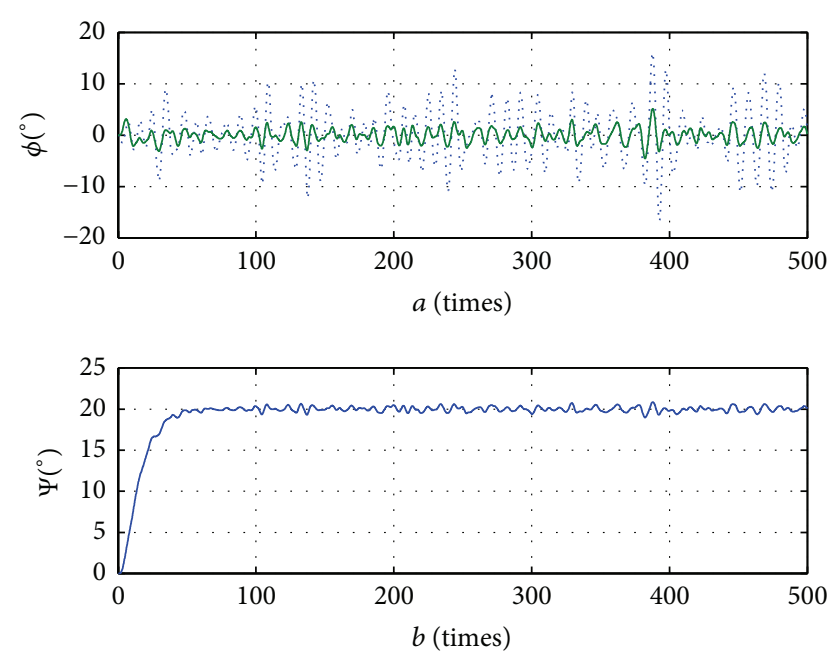

FIGURE 1: Analytic model predictive control on the accurate model.
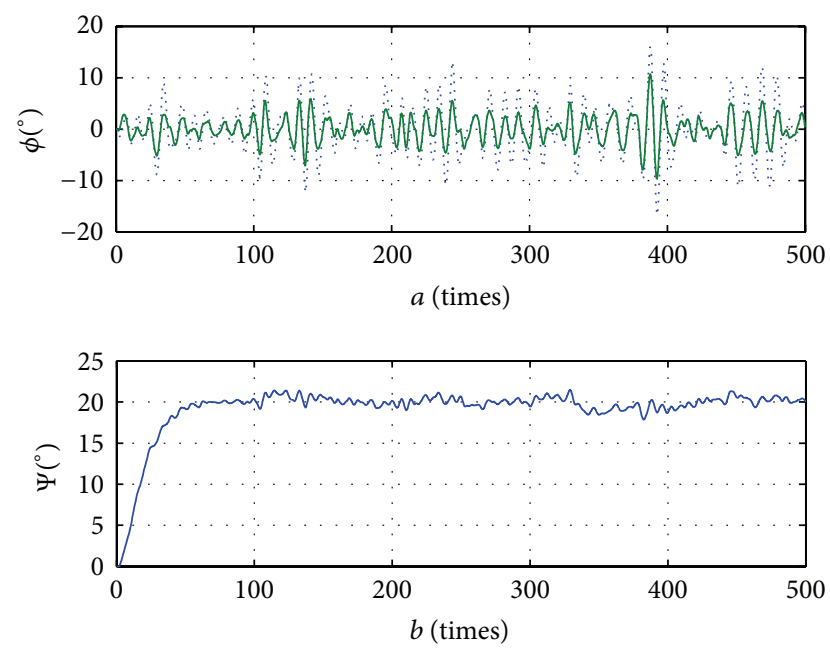

FIGURE 2: Analytic model predictive control on the uncertain model.
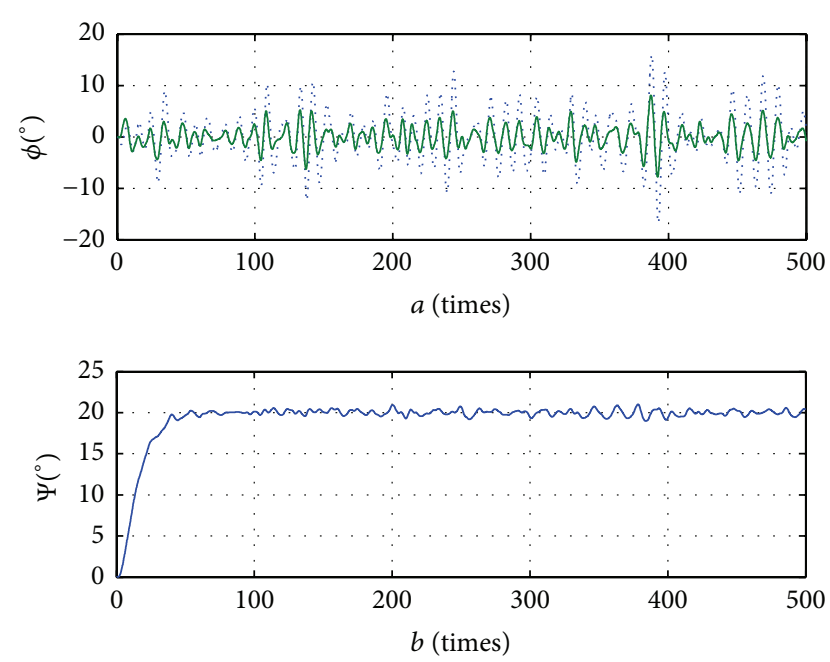

Figure 3: Fuzzy adaptive analytic model predictive control on the uncertain model.
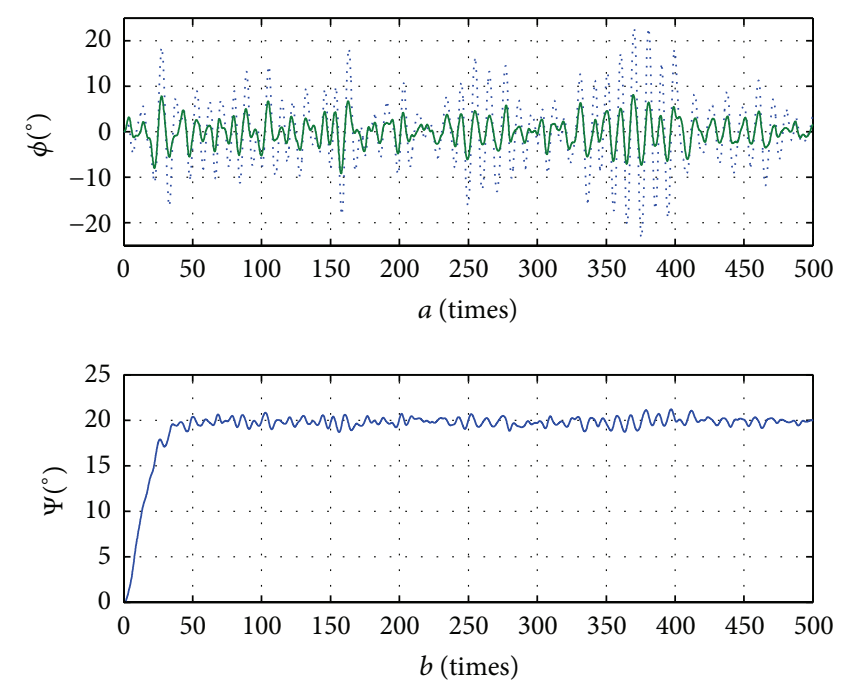

FIGURE 4: Analytic model predictive control on the accurate model.
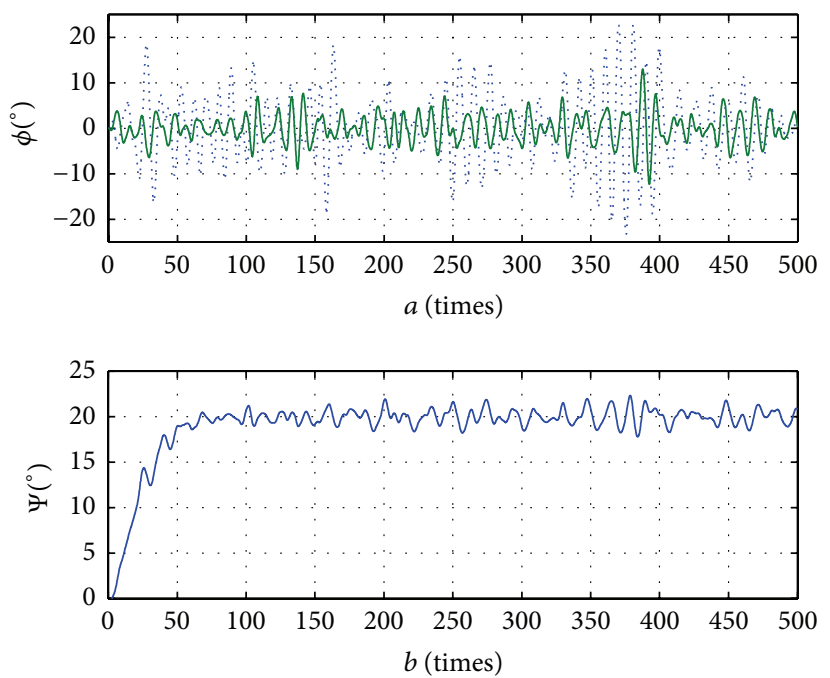

FIGURE 5: Analytic model predictive control on the uncertain model.
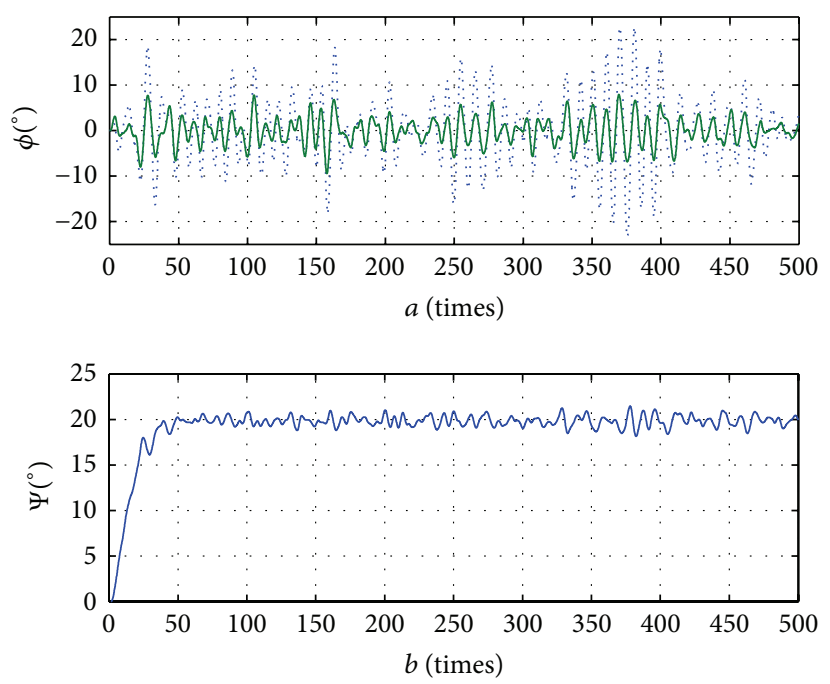

FIGURE 6: Fuzzy adaptive analytic model predictive control on the uncertain model. 
Finally, we apply it to the rudder roll stabilization system control, which is the ship motion model with four freedom degrees. Simulation results show that the proposed algorithm has better control effect and robustness.

\section{Conflict of Interests}

The authors declare that there is no conflict of interests regarding the publication of this paper.

\section{Acknowledgments}

The authors are grateful to the guest editors and anonymous reviewers for their constructive comments based on which the presentation of this paper has been greatly improved.

\section{References}

[1] W. H. Chen, D. J. Ballance, and P. J. Gawthrop, "Optimal control of nonlinear systems: a predictive control approach," Automatica, vol. 39, no. 4, pp. 633-641, 2003.

[2] W.-H. Chen, "Analytic predictive controllers for nonlinear systems with ill-defined relative degree," IEE Proceedings: Control Theory and Applications, vol. 148, no. 1, pp. 9-16, 2001.

[3] X. Z. Lin, L. Xie, and H. Y. Su, "Economic performance for predictive control systems under model uncertainty," Acta Automatica Sinica, vol. 39, no. 7, pp. 1141-1145, 2013.

[4] X.-G. Yan and C. Edwards, "Robust decentralized actuator fault detection and estimation for large-scale systems using a sliding mode observer," International Journal of Control, vol. 81, no. 4, pp. 591-606, 2008.

[5] X.-G. Yan, S. K. Spurgeon, and C. Edwards, "Sliding mode control for time-varying delayed systems based on a reducedorder observer," Automatica, vol. 46, no. 8, pp. 1354-1362, 2010.

[6] X. G. Yan, S. K. Spurgeon, and C. Edwards, "Static output feedback sliding mode control for time-varying delay systems with time-delayed nonlinear disturbances," International Journal of Robust and Nonlinear Control, vol. 20, no. 7, pp. 777-788, 2010.

[7] X. G. Yan and C. Edwards, "Adaptive sliding-mode-observerbased fault reconstruction for nonlinear systems with parametric uncertainties," IEEE Transactions on Industrial Electronics, vol. 55, no. 11, pp. 4029-4036, 2008.

[8] S. Shi, Q. Zhang, and Z. Yuan, "Exponential stability and $L_{2}$ gain analysis for a class of faulty systems," International Journal of Systems Science, vol. 42, no. 3, pp. 377-387, 2011.

[9] S. K. Spurgeon, "Sliding mode observers: a survey," International Journal of Systems Science, vol. 39, no. 8, pp. 751-764, 2008.

[10] Y. Wei, J. Qiu, H. R. Karimi, and M. Wang, "Filtering design for two-dimensional Markovian jump systems with state-delays and deficient mode information," Information Sciences, vol. 269, pp. 316-331, 2014.

[11] Y. Wei, J. Qiu, H. R. Karimi, and M. Wang, "A new design of $H_{\infty}$ filtering for continuous-time Markovian jump systems with time-varying delay and partially accessible mode information," Signal Processing, vol. 93, no. 9, pp. 2392-2407, 2013.

[12] J. H. Park, S. J. Seo, and G. T. Park, "Robust adaptive fuzzy controller for nonlinear system using estimation of bounds for approximation errors," Fuzzy Sets and Systems, vol. 133, no. 1, pp. 19-36, 2003.
[13] J. Qiu, G. Feng, and H. Gao, "Fuzzy-model-based piecewise $H_{\infty}$ static-output-feedback controller design for networked nonlinear systems," IEEE Transactions on Fuzzy Systems, vol. 18, no. 5, pp. 919-934, 2010.

[14] J. Qiu, G. Feng, and H. Gao, “Asynchronous output-feedback control of networked nonlinear systems with multiple packet dropouts: T-S fuzzy affine model-based approach," IEEE Transactions on Fuzzy Systems, vol. 19, no. 6, pp. 1014-1030, 2011.

[15] H.-X. Li and S. C. Tong, "A hybrid adaptive fuzzy control for a class of nonlinear MIMO systems," IEEE Transactions on Fuzzy Systems, vol. 11, no. 1, pp. 24-34, 2003.

[16] C. Wei and Y. Zhang, "Entropy measures for interval-valued intuitionistic fuzzy sets and their application in group decisionmaking," Mathematical Problems in Engineering, vol. 2015, Article ID 563745, 13 pages, 2015.

[17] H. Zhang, J. Hu, and W. Bu, "Research on fuzzy immune selfadaptive PID algorithm based on new Smith predictor for networked control system," Mathematical Problems in Engineering, vol. 2015, Article ID 343416, 6 pages, 2015.

[18] J. Xing and N. Shi, "Adaptive stabilization control for a class of complex nonlinear systems based on T-S fuzzy bilinear model," Mathematical Problems in Engineering, vol. 2015, Article ID 659521, 11 pages, 2015.

[19] G. Wang, Generalized Inverse of Matrices and Operators, Science Press, 1998.

[20] T. Perez, Ship Motion Control: Course Keeping and Roll Stabilisation Using Rudder and Fins, Springer, 2005. 


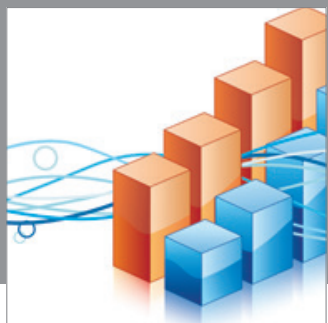

Advances in

Operations Research

mansans

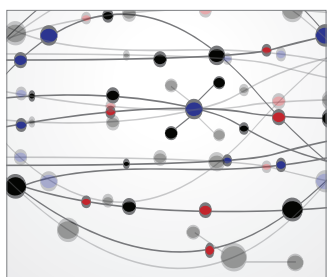

The Scientific World Journal
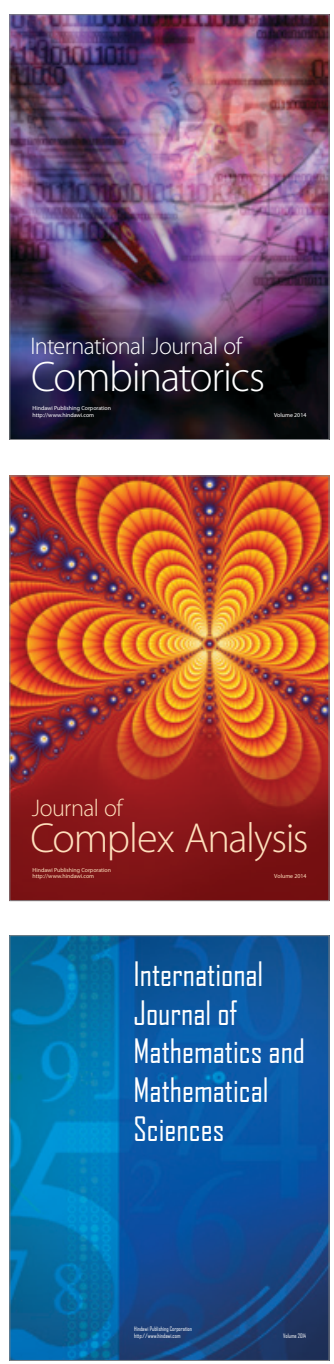
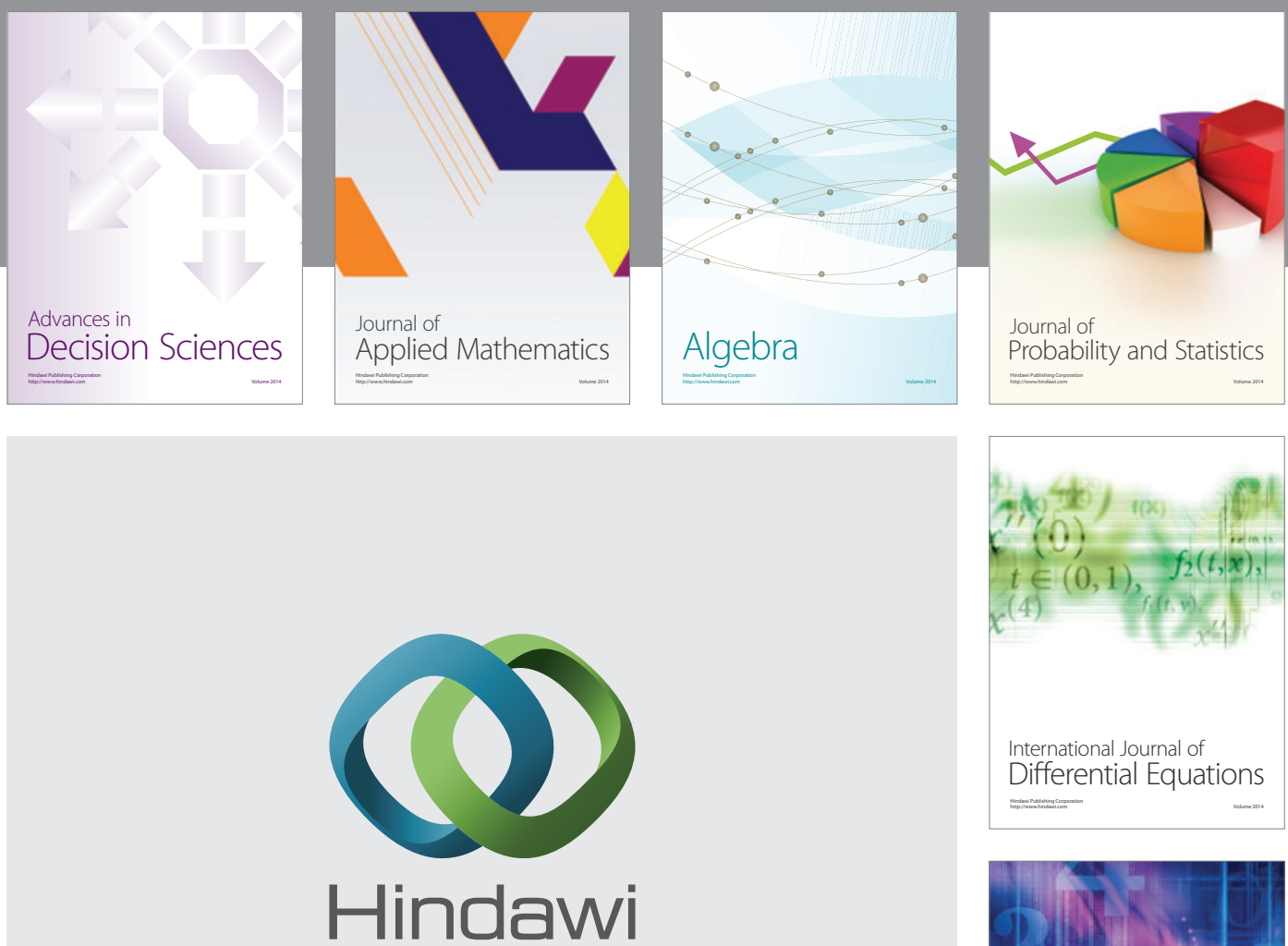

Submit your manuscripts at http://www.hindawi.com
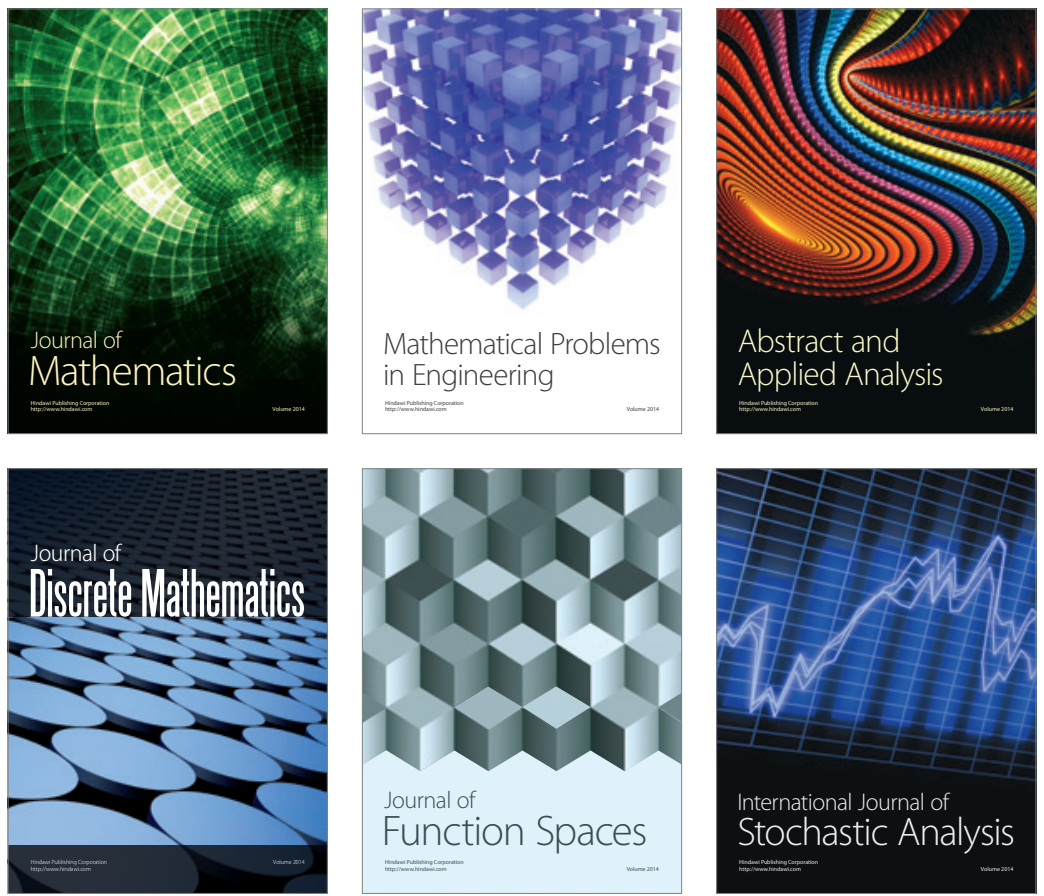

Journal of

Function Spaces

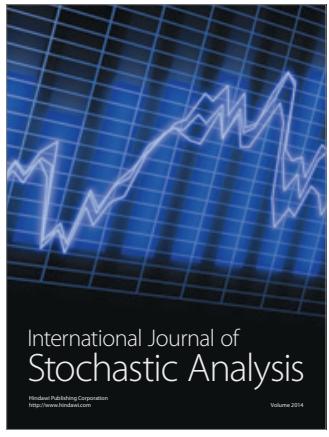

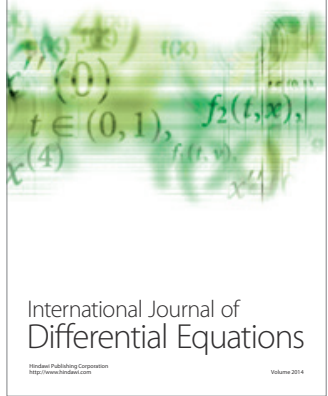
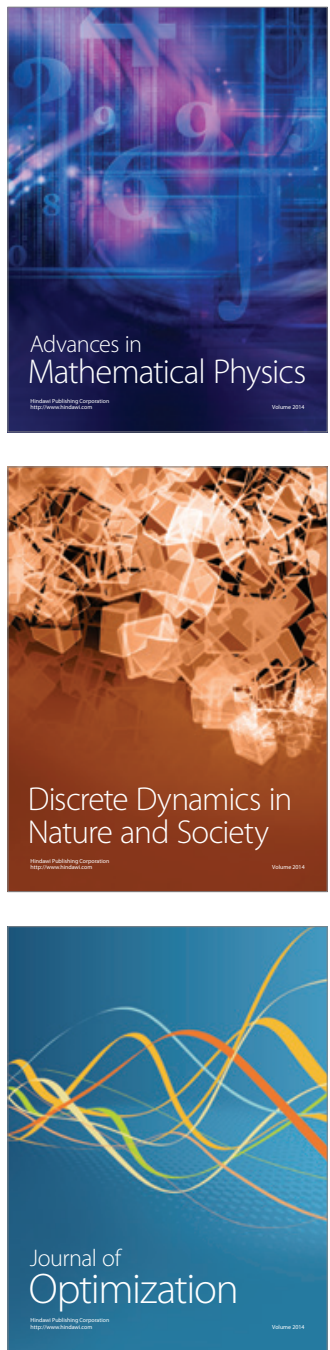